\title{
Linear Boundary Value Problems for Normally Solvable Operator Equations in a Banach Space
}

\author{
A. M. Samoilenko, A. A. Boichuk, and V. F. Zhuravlev \\ Institute for Mathematics, National Academy of Sciences, Kyiv, Ukraine \\ e-mail:sam@imath.kiev.ua,boichuk@imath.kiev.ua
}

Received December 31, 2012

\begin{abstract}
We consider linear boundary value problems for operator equations with generalized-invertible operator in a Banach or Hilbert space. We obtain solvability conditions for such problems and indicate the structure of their solutions. We construct a generalized Green operator and analyze its properties and the relationship with a generalized inverse operator of the linear boundary value problem. The suggested approach is illustrated in detail by an example.
\end{abstract}

DOI: $10.1134 /$ S0012266114030057

The classical theory of periodic problems for various classes of differential equations [1-3] arose well before the development of methods of functional analysis. Its further development [4-8] used functional analysis as a technique for studying general boundary value problems. For example, on the basis of the theory of the generalized inversion of matrices [9], methods of the investigation of periodic boundary value problems were extended to general boundary value problems for various classes of functional-differential equations: ordinary differential systems in critical (resonance) cases [10]; differential systems with retarded argument [11]; ordinary impulsive differential systems $[12,13]$. General theorems on the solvability and the representation of solutions of critical boundary value problems were proved for various classes of linear and nonlinear equations, and spaces in which these boundary value problems were considered were generalized.

Further development was given by the theory of boundary value problems for ordinary differential equations in a Banach space with the finite-dimensional Euclidean space of values of the unknown function replaced by a more general Banach space [14]. For example, a criterion for the existence of solutions of linear boundary value problems was obtained in [15] for ordinary differential equations in a Banach space in the critical case. Conditions for the existence of periodic solutions of differential and difference equations in the Banach space $\mathbf{m}$ of bounded numerical sequences were considered in the monograph [16, p. 266]. Conditions for the existence and bifurcation of bounded, on the entire real line $\mathbb{R}=(-\infty,+\infty)$, solutions of a weakly perturbed differential equation in a Banach space were obtained in [17].

From the viewpoint of the theory of operators in function spaces, the above-listed boundary value problems have the following specific features: the original equations in these boundary value problems have solutions for an arbitrary right-hand side. In S.G. Krein's terminology [18, p. 8], such problems are everywhere solvable. However, there exist boundary value problems for which the original operator equation is not everywhere solvable, for example, problems for integro-differential equations [19] and problems for singular differential systems [20-22]. In this connection, it is topical to study general boundary value problems for operator equations in a Banach space that are not everywhere solvable, and this is what we deal with in the present paper.

\section{STATEMENT OF THE PROBLEM}

Let $\mathbf{l}_{\infty}\left(\mathcal{I}, \mathbf{B}_{1}\right)$ be the Banach space of bounded vector functions $z(t)$ defined on a finite interval $\mathcal{I}$ and ranging in some Banach space $\mathbf{B}_{1}, z(\cdot): \mathcal{I} \rightarrow \mathbf{B}_{1}$ with norm \|\|$z\|\|=\sup _{t \in \mathcal{I}}\|z(t)\|_{\mathbf{B}_{1}}$, let $\mathbf{l}_{\infty}\left(\mathcal{I}, \mathbf{B}_{2}\right)$ be the Banach space of bounded vector functions $\varphi(t)$ defined on the same interval $\mathcal{I}$ and 
ranging in some Banach space $\mathbf{B}_{2}$ with norm $\||\varphi|\|=\sup _{t \in \mathcal{I}}\|\varphi(t)\|_{\mathbf{B}_{2}}$, and let $\mathbf{B}$ be the Banach space of vectors with constant components. The notation of function spaces has been borrowed from the monograph [23].

Let $L: \mathbf{l}_{\infty}\left(\mathcal{I}, \mathbf{B}_{1}\right) \rightarrow \mathbf{l}_{\infty}\left(\mathcal{I}, \mathbf{B}_{2}\right)$ be a linear bounded generalized-invertible operator, and let $\ell=\operatorname{col}\left(l_{1}, l_{2}, l_{3}, \ldots\right): \mathbf{l}_{\infty}\left(\mathcal{I}, \mathbf{B}_{1}\right) \rightarrow \mathbf{B}$ be a linear bounded vector functional. The generalized invertibility of the operator $L$ is defined as follows [24, p. 139]: the operator $L$ is normally solvable, and its null space $N(L)$ and range $R(L)$ are complemented subspaces of the Banach spaces $\mathbf{l}_{\infty}\left(\mathcal{I}, \mathbf{B}_{1}\right)$ and $\mathbf{l}_{\infty}\left(\mathcal{I}, \mathbf{B}_{2}\right)$, respectively. In this case, there exist [25] bounded projections $\mathcal{P}_{N(L)}: \mathbf{l}_{\infty}\left(\mathcal{I}, \mathbf{B}_{1}\right) \rightarrow$ $N(L)$ and $\mathcal{P}_{Y_{L}}: \mathbf{l}_{\infty}\left(\mathcal{I}, \mathbf{B}_{2}\right) \rightarrow Y_{L}$, which decompose the spaces $\mathbf{l}_{\infty}\left(\mathcal{I}, \mathbf{B}_{1}\right)$ and $\mathbf{l}_{\infty}\left(\mathcal{I}, \mathbf{B}_{2}\right)$ into direct sums of closed subspaces,

$$
\mathbf{l}_{\infty}\left(\mathcal{I}, \mathbf{B}_{1}\right)=N(L) \oplus X_{L}, \quad \mathbf{l}_{\infty}\left(\mathcal{I}, \mathbf{B}_{2}\right)=Y_{L} \oplus R(L)
$$

Traditionally, generalized-invertible operators include the following operators: Fredholm operators of index zero $\left(\operatorname{dim} N(L)=\operatorname{dim} Y_{L}<\infty\right)$ [26]; Fredholm operators $(\operatorname{dim} N(L)=n<\infty$, $\operatorname{dim} Y_{L}=d<\infty$, and $\left.n \neq d\right)$ [27], where Fredholm theory was generalized to singular integral equations; $n$-normal operators $\left[\operatorname{dim} N(L)=n<\infty\right.$ and $\operatorname{dim} Y_{L}=\infty$ ] with complemented range; $d$-normal operators $\left(\operatorname{dim} N(L)=\infty\right.$ and $\left.\operatorname{dim} Y_{L}=d<\infty\right)$ with complemented kernel [18, pp. 27, 31]; topologically Fredholm operators of index zero $\left[\operatorname{dim} N(L)=\infty, \operatorname{dim} Y_{L}=\infty\right.$, and $N(L)$ is isomorphic to $Y_{L}$ ] (reducible-invertible operators if the operator maps a Banach space into itself $\left[28\right.$, p. 24]); topologically Fredholm operators $\left[\operatorname{dim} N(L)=\infty\right.$ and $\left.\operatorname{dim} Y_{L}=\infty\right]$ [29].

Note that Fredholm operators of index zero and Fredholm operators are distinguished in Russian publications $[11,13,18,23]$ (where they are called Fredholm and Noether operators, respectively), and both classes are referred to as Fredholm operators in English publications. In foreign publications, $n$ - and $d$-normal operators are referred to as semi-Fredholm or semi-Noether operators among which $\Phi_{+}$-operators $[\operatorname{dim} N(L)<\infty]$ and $\Phi_{-}$-operators $\left(\operatorname{dim} Y_{L}<\infty\right)$ are distinguished [30].

In the present paper, we obtain solvability conditions for general boundary value problems for operator equations with generalized-invertible operators in abstract Banach spaces and analyze the properties of the generalized Green operator for such boundary value problems.

\section{BOUNDARY VALUE PROBLEM}

Let us study necessary and sufficient solvability conditions and the structure of the set of solutions $z(t) \in \mathbf{l}_{\infty}\left(\mathcal{I}, \mathbf{B}_{1}\right)$ for the linear boundary value problem

$$
\begin{aligned}
(L z)(t) & =\varphi(t), \\
\ell z(\cdot) & =\alpha .
\end{aligned}
$$

The normally solvable equation (1) is solvable if and only if $\varphi(t) \in \mathbf{l}_{\infty}\left(\mathcal{I}, \mathbf{B}_{2}\right)$ satisfies the condition $[18$, p. $14 ; 24$, p. 133$]$

$$
\left(\mathcal{P}_{Y_{L}} \varphi\right)(t)=0
$$

where $\mathcal{P}_{Y_{L}}: \mathbf{l}_{\infty}\left(\mathcal{I}, \mathbf{B}_{2}\right) \rightarrow Y_{L} \subset \mathbf{l}_{\infty}\left(\mathcal{I}, \mathbf{B}_{2}\right)$ is the projection onto the subspace $Y_{L}$ isomorphic to the null space $N\left(L^{*}\right)$ of the operator $L^{*}$ adjoint to the operator $L$.

Under condition (3), the general solution of Eq. (1) can be represented in the form

$$
z(t)=\left(\mathcal{P}_{N(L)} \hat{z}\right)(t)+\left(L^{-} \varphi\right)(t),
$$

where $\hat{z}(t)$ is an arbitrary element of the space $\mathbf{l}_{\infty}\left(\mathcal{I}, \mathbf{B}_{1}\right)$ and $L^{-}$is a bounded generalized inverse of $L$ [11, p. $53 ; 13$, p. 40; 31].

The solution (4) of the inhomogeneous operator equation (1) is a solution of the boundary value problem (1), (2) if and only if the element $\hat{z}(t)=\hat{z}_{0}(t) \in \mathbf{l}_{\infty}\left(\mathcal{I}, \mathbf{B}_{1}\right)$ satisfies the operator equation

$$
\ell\left(\mathcal{P}_{N(L)} \hat{z}_{0}\right)(\cdot)+\ell\left(L^{-} \varphi\right)(\cdot)=\alpha,
$$

which is obtained by the substitution of the solution (4) into the boundary condition (2). 
By $\mathcal{L} *=\ell \mathcal{P}_{N(L)} *$ we denote the linear operator mapping the Banach space $\mathbf{l}_{\infty}\left(\mathcal{I}, \mathbf{B}_{1}\right)$ into the Banach space $\mathbf{B}$. The operator $\mathcal{L}$ is bounded, because it is the composition of the bounded functional $\ell$ and the bounded projection $\mathcal{P}_{N(L)}$.

Let the operator $\mathcal{L}$ belong to the class of generalized-invertible operators; i.e., the null space $N(\mathcal{L})$ and the range $R(\mathcal{L})$ of the operator $\mathcal{L}$ are complemented in the Banach spaces $\mathbf{l}_{\infty}\left(\mathcal{I}, \mathbf{B}_{1}\right)$ and $\mathbf{B}$, respectively.

By $\mathcal{P}_{N(\mathcal{L})}: \mathbf{l}_{\infty}\left(\mathcal{I}, \mathbf{B}_{1}\right) \rightarrow N(\mathcal{L})$ we denote the bounded projection of the Banach space $\mathbf{l}_{\infty}\left(\mathcal{I}, \mathbf{B}_{1}\right)$ onto the null space of the operator $\mathcal{L}$, by $\mathcal{P}_{Y_{\mathcal{L}}}: \mathbf{B} \rightarrow Y_{\mathcal{L}}$ we denote the bounded projection of the Banach space $\mathbf{B}$ onto the subspace $Y_{\mathcal{L}} \subset \mathbf{B}$, and by $\mathcal{L}^{-}$we denote a linear bounded generalizedinverse operator of the operator $\mathcal{L}$.

From the operator equation

$$
\left(\mathcal{L} \hat{z}_{0}\right)(\cdot)=\alpha-\ell\left(L^{-} \varphi\right)(\cdot)
$$

we find an element $\hat{z}_{0}(t) \in \mathbf{l}_{\infty}\left(\mathcal{I}, \mathbf{B}_{1}\right)$ for which a the solution (4) of Eq. (1) existing under condition (3) is a solution of the boundary value problem (1), (2). Since, by assumption, the operator $\mathcal{L}$ is generalized-invertible and hence normally solvable, it follows that Eq. (5) is solvable if and only if

$$
\mathcal{P}_{Y_{\mathcal{L}}}\left\{\alpha-\ell\left(L^{-} \varphi\right)(\cdot)\right\}=0 .
$$

Under this condition, Eq. (5) has the family of solutions

$$
\hat{z}_{0}(t)=\left(\mathcal{P}_{N(\mathcal{L})} \hat{z}\right)(t)+\left(\mathcal{L}^{-}\left\{\alpha-\ell\left(L^{-} \varphi\right)(\cdot)\right\}\right)(t),
$$

where $\hat{z}(t)$ is an arbitrary element of the Banach space $\mathbf{l}_{\infty}\left(\mathcal{I}, \mathbf{B}_{1}\right)$.

By substituting $\hat{z}_{0}(t)(7)$ for $\hat{z}(t)$ into (4), we obtain the general solution of the boundary value problem (1), (2) in the form

$$
\begin{aligned}
z(t) & =\left(\mathcal{P}_{N(L)}\left\{\left(\mathcal{P}_{N(\mathcal{L})} \hat{z}\right)(\cdot)+\mathcal{L}^{-}\left[\alpha-\ell\left(L^{-} \varphi\right)(\cdot)\right]\right\}\right)(t)+\left(L^{-} \varphi\right)(t) \\
& =\left(\mathcal{P}_{N(L)} \mathcal{P}_{N(\mathcal{L})} \hat{z}\right)(t)+(G \varphi)(t)+\left(\mathcal{P}_{N(L)} \mathcal{L}^{-} \alpha\right)(t) .
\end{aligned}
$$

Here $\mathcal{P}_{N(L)} \mathcal{P}_{N(\mathcal{L})}$ is the resolving operator $[14$, p. 147] of the homogeneous boundary value problem corresponding to $(1),(2)$, and $G: \mathbf{l}_{\infty}\left(\mathcal{I}, \mathbf{B}_{2}\right) \rightarrow N(\ell) \subset \mathbf{l}_{\infty}\left(\mathcal{I}, \mathbf{B}_{1}\right)$ is the operator given by the formula

$$
(G \varphi)(t)=\left(L^{-} \varphi\right)(t)-\left(\mathcal{P}_{N(L)} \mathcal{L}^{-} \ell\left(L^{-} \varphi\right)(\cdot)\right)(t)
$$

and is called the generalized Green operator of the semihomogeneous $(\alpha=0)$ boundary value problem (1), (2).

We have thereby proved the following assertion.

Theorem 1. Let $L: \mathbf{l}_{\infty}\left(\mathcal{I}, \mathbf{B}_{1}\right) \rightarrow \mathbf{l}_{\infty}\left(\mathcal{I}, \mathbf{B}_{2}\right)$ and $\mathcal{L}: \mathbf{l}_{\infty}\left(\mathcal{I}, \mathbf{B}_{1}\right) \rightarrow \mathbf{B}$ be generalized-invertible operators. Then the homogeneous boundary value problem corresponding to $(1),(2)[\varphi(t)=0$, $\alpha=0]$ has the family of linearly independent solutions

$$
z(t)=\left(\mathcal{P}_{N(L)} \mathcal{P}_{N(\mathcal{L})} \hat{z}\right)(t),
$$

where $\hat{z}(t)$ is an arbitrary element of the Banach space $\mathbf{l}_{\infty}\left(\mathcal{I}, \mathbf{B}_{1}\right)$.

The inhomogeneous boundary value problem (1), (2) is solvable if and only if $\varphi(t) \in \mathbf{l}_{\infty}\left(\mathcal{I}, \mathbf{B}_{2}\right)$ and $\alpha \in \mathbf{B}$ satisfy conditions (3) and (6) and in this case has a family of solutions of the form (8),

$$
z(t)=\left(\mathcal{P}_{N(L)} \mathcal{P}_{N(\mathcal{L})} \hat{z}\right)(t)+(G \varphi)(t)+\left(\mathcal{P}_{N(L)} \mathcal{L}^{-} \alpha\right)(t),
$$

where $\mathcal{P}_{N(L)} \mathcal{P}_{N(\mathcal{L})}$ is the resolving operator of the homogeneous boundary value problem corresponding to (1), (2) and $G$ is the generalized Green operator (9).

Corollary 1. If the operators $L$ and $\mathcal{L}$ are normally solvable and act in Hilbert spaces, then in Theorem 1, one should replace the generalized-inverse operators $L^{-}$and $\mathcal{L}^{-}$by the pseudoinverse operators $L^{+}$and $\mathcal{L}^{+}[13]$ and the projections $\mathcal{P}_{N(L)}, \mathcal{P}_{Y_{L}}$ and $\mathcal{P}_{N(\mathcal{L})}, \mathcal{P}_{Y_{\mathcal{L}}}$ by the orthogonal 
projections $P_{N(L)}, P_{N\left(L^{*}\right)}$ and $P_{N(\mathcal{L})}, P_{N\left(\mathcal{L}^{*}\right)}$, respectively. In this case, formula (10) acquires the form

$$
z(t)=\left(P_{N(L)} P_{N(\mathcal{L})} \hat{z}\right)(t)+(G \varphi)(t)+\left(P_{N(L)} \mathcal{L}^{+} \alpha\right)(t),
$$

where $(G \varphi)(t)=\left(L^{+} \varphi\right)(t)-\left(P_{N(L)} \mathcal{L}^{+} \ell\left(L^{+} \varphi\right)(\cdot)\right)(t)$ is the generalized Green operator.

Remark 1. When considering linear boundary value problems of the form (1), (2) for the everywhere solvable differential operator $L z(t)=z^{\prime}(t)-A(t) z(t)$ that acts from the Banach space $\mathbf{C}^{1}(\mathcal{I}, \mathbf{B})$ of continuously differentiable functions ranging in the Banach space $\mathbf{B}$ into the Banach space $\mathbf{C}(\mathcal{I}, \mathbf{B})$ of continuous vector functions with supremum norm, we obtain the well-known results in [15].

Remark 2. By considering periodic boundary value problems for linear difference equations in the Banach space $\mathbf{m}$, we obtain the earlier-known results obtained in [16] in the resonance-free case.

As a corollary of Theorem 1, consider the Cauchy problem

$$
\begin{aligned}
(L z)(t) & =\varphi(t), \\
\ell z(\cdot) & \equiv z\left(t_{0}\right)=z_{0}, \quad t_{0} \in \mathcal{I},
\end{aligned}
$$

which is a specific boundary value problem with $\mathcal{L} *=\left(\mathcal{P}_{N(L)} *\right)\left(t_{0}\right)$.

Corollary 2. Let $L$ and $\mathcal{L}$ be generalized-invertible operators. Then the Cauchy problem (11), (12) is solvable if and only if $z_{0}=z\left(t_{0}\right) \in \mathbf{B}_{1}$ and $\varphi(t) \in \mathbf{l}_{\infty}\left(\mathcal{I}, \mathbf{B}_{2}\right)$ satisfy the conditions

$$
\left(\mathcal{P}_{Y_{L}} \varphi\right)(t)=0, \quad \mathcal{P}_{Y_{\mathcal{L}}}\left\{z_{0}-\left(L^{-} \varphi\right)\left(t_{0}\right)\right\}=0
$$

and in this case has the family of solutions

$$
z(t)=\left(\mathcal{P}_{N(L)} \mathcal{P}_{N(\mathcal{L})} \hat{z}\right)(t)+\left(G_{0} \varphi\right)(t)+\left(\mathcal{P}_{N(L)} \mathcal{L}^{-} z_{0}\right)(t)
$$

where $\mathcal{P}_{N(L)} \mathcal{P}_{N(\mathcal{L})}$ is the resolving operator of the homogeneous Cauchy problem $\left[z_{0}=0, \varphi(t)=0\right]$ corresponding to (11), (12), $\hat{z}(t)$ is an arbitrary element of the Banach space $\mathbf{l}_{\infty}\left(\mathcal{I}, \mathbf{B}_{1}\right)$, and $\left(G_{0} \varphi\right)(t)=\left(L^{-} \varphi\right)(t)-\left(\mathcal{P}_{N(L)} \mathcal{L}^{-}\left(L^{-} \varphi\right)\left(t_{0}\right)\right)(t)$ is the Green operator of the semihomogeneous $\left(z_{0}=0\right)$ Cauchy problem (11), (12).

Remark 3. If $L$ is an everywhere solvable differential operator $(L z)(t)=z^{\prime}(t)-A(t) z(t)$ acting from the Banach space $\mathbf{C}^{1}(\mathcal{I}, \mathbf{B})$ of continuously differentiable functions ranging in a Banach space $\mathbf{B}$ into the Banach space $\mathbf{C}(\mathcal{I}, \mathbf{B})$ of continuous vector functions with the supremum norm and $\varphi(t)$ is a continuous vector function on the interval $\mathcal{I}$, then $\mathcal{P}_{Y_{L}} \equiv 0$, the operator $\mathcal{L} *=\left(\mathcal{P}_{N(L)} *\right)\left(t_{0}\right)$ is invertible for each $t_{0} \in \mathcal{I}$, and the generalized-invertible operator $L^{-}$is the integral right inverse operator $L_{r}^{-1}$. In this case, formula (13) acquires the form [14, p. 148]

$$
z(t)=U\left(t, t_{0}\right) z_{0}+\int_{t_{0}}^{t} U(t, \tau) \varphi(\tau) d \tau, \quad t_{0} \in \mathcal{I}
$$

where $U(t, \tau)=U(t) U^{-1}(\tau)$ is the evolution operator.

\section{GENERALIZED GREEN OPERATOR AND ITS PROPERTIES}

By using the approach suggested in [32], we rewrite the boundary value problem (1), (2) in the vector-matrix form

$$
(\Lambda z)(t)=f(t)
$$

where $\Lambda=\operatorname{col}[L, \ell]$ is a linear operator and $f(t)=\operatorname{col}[\varphi(t), \alpha] \in \mathbf{l}_{\infty}\left(\mathcal{I}, \mathbf{B}_{2}\right) \times \mathbf{B}$. 
The operator $\Lambda: \mathbf{l}_{\infty}\left(\mathcal{I}, \mathbf{B}_{1}\right) \rightarrow \mathbf{l}_{\infty}\left(\mathcal{I}, \mathbf{B}_{2}\right) \times \mathbf{B}$ is bounded if the norm on the space $\mathbf{l}_{\infty}\left(\mathcal{I}, \mathbf{B}_{2}\right) \times \mathbf{B}$ is introduced as follows:

$$
\|(\varphi, \alpha)\|_{\mathbf{l}_{\infty}\left(\mathcal{I}, \mathbf{B}_{2}\right) \times \mathbf{B}}=\|\varphi\|_{\mathbf{l}_{\infty}\left(\mathcal{I}, \mathbf{B}_{2}\right)}+\|\alpha\|_{\mathbf{B}}, \quad \varphi \in \mathbf{l}_{\infty}\left(\mathcal{I}, \mathbf{B}_{2}\right), \quad \alpha \in \mathbf{B} .
$$

Consider some properties of the generalized Green operator (9) $G: \mathbf{l}_{\infty}\left(\mathcal{I}, \mathbf{B}_{2}\right) \rightarrow \operatorname{ker} \ell \subset$ $\mathbf{l}_{\infty}\left(\mathcal{I}, \mathbf{B}_{1}\right)$ and its relationship with the operator $\Lambda$. To simplify the notation, throughout the following we omit parentheses and variables.

Lemma 1. The generalized Green operator (9) satisfies the relation

$$
\Lambda G=\left[\begin{array}{c}
I_{1_{\infty}\left(\mathcal{I}, \mathbf{B}_{2}\right)}-\mathcal{P}_{Y_{L}} \\
\mathcal{P}_{Y_{\mathcal{L}}} \ell L^{-}
\end{array}\right]
$$

Proof. Indeed, by substituting the expression (9) for $G$ into (14), we obtain

$$
\begin{aligned}
\Lambda G & =\left[\begin{array}{c}
L \\
\ell
\end{array}\right]\left[L^{-}-\mathcal{P}_{N(L)} \mathcal{L}^{-} \ell L^{-}\right]=\left[\begin{array}{c}
L\left(L^{-}-\mathcal{P}_{N(L)} \mathcal{L}^{-} \ell L^{-}\right) \\
\ell\left(L^{-}-\mathcal{P}_{N(L)} \mathcal{L}^{-} \ell L^{-}\right)
\end{array}\right] \\
& =\left[\begin{array}{c}
L L^{-}-L \mathcal{P}_{N(L)} \mathcal{L}^{-} \ell L^{-} \\
\ell L^{-}-\ell \mathcal{P}_{N(L)} \mathcal{L}^{-} \ell L^{-}
\end{array}\right]=\left[\begin{array}{c}
L L^{-} \\
\left(I_{\mathrm{B}}-\mathcal{L} \mathcal{L}^{-}\right) \ell L^{-}
\end{array}\right]=\left[\begin{array}{c}
I_{\mathbf{l}_{\infty}\left(\mathcal{I}, \mathbf{B}_{2}\right)}-\mathcal{P}_{Y_{L}} \\
\mathcal{P}_{Y_{\mathcal{L}}} \ell L^{-}
\end{array}\right],
\end{aligned}
$$

because $L \mathcal{P}_{N(L)}=0, L L^{-}=I_{1_{\infty}\left(\mathcal{I}, \mathbf{B}_{2}\right)}-\mathcal{P}_{Y_{L}}$, and $I_{\mathbf{B}}-\mathcal{L} \mathcal{L}^{-}=\mathcal{P}_{Y_{\mathcal{L}}}[11$, p. $53 ; 13$, p. 40]. form

Remark 4. In the case of an everywhere solvable equation $L z=\varphi$, relation (14) acquires the

$$
\Lambda G=\left[\begin{array}{c}
I_{\mathbf{l}_{\infty}\left(\mathcal{I}, \mathbf{B}_{2}\right)} \\
\mathcal{P}_{Y_{\mathcal{L}}} \ell L_{r}^{-1}
\end{array}\right],
$$

because $R(L)=\mathbf{l}_{\infty}\left(\mathcal{I}, \mathbf{B}_{2}\right), \mathcal{P}_{Y_{L}} \equiv 0$, and $L^{-}=L_{r}^{-1}$.

Theorem 2. Let $L: \mathbf{l}_{\infty}\left(\mathcal{I}, \mathbf{B}_{1}\right) \rightarrow \mathbf{l}_{\infty}\left(\mathcal{I}, \mathbf{B}_{2}\right)$ and $\mathcal{L}: \mathbf{l}_{\infty}\left(\mathcal{I}, \mathbf{B}_{1}\right) \rightarrow \mathbf{B}$ be generalized-invertible operators. Then the operator

$$
\Lambda^{-}=\left[G, \mathcal{P}_{N(L)} \mathcal{L}^{-}\right]
$$

is a bounded generalized inverse operator of the bounded linear operator $\Lambda=\operatorname{col}[L, \ell]$.

Proof. As was shown above, if conditions (3) and (6) are satisfied, then the boundary value problem

$$
\Lambda z=\left[\begin{array}{l}
L \\
\ell
\end{array}\right] z=\left[\begin{array}{l}
\varphi \\
\alpha
\end{array}\right]
$$

is solvable and its solution has the form (10). Then for the generalized-inverse operator

$$
\Lambda^{-}: \mathbf{l}_{\infty}\left(\mathcal{I}, \mathbf{B}_{2}\right) \times \mathbf{B} \rightarrow \mathbf{l}_{\infty}\left(\mathcal{I}, \mathbf{B}_{1}\right)
$$

we have the representation

$$
\Lambda^{-} f=\left[\begin{array}{c}
L \\
\ell
\end{array}\right]^{-}\left[\begin{array}{l}
\varphi \\
\alpha
\end{array}\right]=G \varphi+\mathcal{P}_{N(L)} \mathcal{L}^{-} \alpha .
$$

Let us show that the operator $\Lambda^{-}$has the following properties.

1. $\Lambda^{-} \Lambda \Lambda^{-}=\Lambda^{-}$.

2. $\Lambda \Lambda^{-} \Lambda=\Lambda$.

DIFFERENTIAL EQUATIONS $\quad$ Vol. $50 \quad$ No. $3 \quad 2014$

(Reg. No. 305, 19.3.2014) 
These properties define a generalized-inverse operator. As was mentioned in [24, p. 140], the second property is a consequence of the first one. Therefore, to prove the theorem it suffices to show that the operator $\Lambda^{-}$satisfies the relation $\Lambda^{-} \Lambda \Lambda^{-}=\Lambda^{-}$. Since $L \mathcal{P}_{N(L)} \equiv 0$ and $\ell \mathcal{P}_{N(L)}=\mathcal{L}$, we have

$$
\Lambda \Lambda^{-}=\left[\begin{array}{c}
L \\
\ell
\end{array}\right]\left[G, \mathcal{P}_{N(L)} \mathcal{L}^{-}\right]=\left[\begin{array}{cc}
L G & L \mathcal{P}_{N(L)} \mathcal{L}^{-} \\
\ell G & \ell \mathcal{P}_{N(L)} \mathcal{L}^{-}
\end{array}\right]=\left[\begin{array}{cc}
I_{1_{\infty}\left(\mathcal{I}, \mathbf{B}_{2}\right)}-\mathcal{P}_{Y_{L}} & 0 \\
\mathcal{P}_{Y_{\mathcal{L}}} \ell L^{-} & \mathcal{L} \mathcal{L}^{-}
\end{array}\right]
$$

It follows from the relations $L^{-} \mathcal{P}_{Y_{L}}=0, \mathcal{L}^{-} \mathcal{P}_{Y_{\mathcal{L}}}=0$, and $G \mathcal{P}_{Y_{L}}=0$ that

$$
\begin{aligned}
\Lambda^{-} \Lambda \Lambda^{-} & =\left[G, \mathcal{P}_{N(L)} \mathcal{L}^{-}\right]\left[\begin{array}{cc}
I_{1_{\infty}\left(\mathcal{I}, \mathbf{B}_{2}\right)}-\mathcal{P}_{Y_{L}} & 0 \\
\mathcal{P}_{Y_{\mathcal{L}}} \ell L^{-} & \mathcal{L} \mathcal{L}^{-}
\end{array}\right] \\
& =\left[G\left(I_{1_{\infty}\left(\mathcal{I}, \mathbf{B}_{2}\right)}-\mathcal{P}_{Y_{L}}\right)+\mathcal{P}_{N(L)} \mathcal{L}^{-} \mathcal{P}_{Y_{\mathcal{L}}} \ell L^{-}, \mathcal{P}_{N(L)} \mathcal{L}^{-} \mathcal{L} \mathcal{L}^{-}\right] \\
& =\left[G-G \mathcal{P}_{Y_{L}}+\mathcal{P}_{N(L)} \mathcal{L}^{-} \mathcal{P}_{Y_{\mathcal{L}}} \ell L^{-}, \mathcal{P}_{N(L)} \mathcal{L}^{-}\right]=\left[G, \mathcal{P}_{N(L)} \mathcal{L}^{-}\right]
\end{aligned}
$$

The boundedness of the operator $\Lambda^{-}$follows from the boundedness of the operators $L^{-}, G$, and $\mathcal{P}_{N(L)} \mathcal{L}^{-}$. Therefore, the operator $\Lambda^{-}: \mathbf{l}_{\infty}\left(\mathcal{I}, \mathbf{B}_{2}\right) \times \mathbf{B} \rightarrow \mathbf{l}_{\infty}\left(\mathcal{I}, \mathbf{B}_{1}\right)$ is a bounded generalized inverse of the operator $\Lambda$ of the original boundary value problem.

Theorem 3. The solvability conditions (3) and (6) for the boundary value problem (1), (2) are equivalent to the condition

$$
\mathcal{P}_{Y_{\Lambda}} f=0,
$$

where $\mathcal{P}_{Y_{\Lambda}}$ is the projection onto the null space $N(\Lambda)$ of the operator $\Lambda$.

Proof. By using the formula $\left[11\right.$, p. 53; 13, p. 40] $\Lambda \Lambda^{-}=I_{1_{\infty}\left(\mathcal{I}, \mathbf{B}_{2}\right) \times \mathbf{B}}-\mathcal{P}_{Y_{\Lambda}}$, which relates the generalized inverse operator $\Lambda^{-}$and the projection $\mathcal{P}_{Y_{\Lambda}}$, and relation (16), we find the projection $\mathcal{P}_{Y_{\Lambda}}$, which has the block structure

$$
\begin{aligned}
\mathcal{P}_{Y_{\Lambda}} & =I_{\mathbf{l}_{\infty}\left(\mathcal{I}, \mathbf{B}_{2}\right) \times \mathbf{B}}-\Lambda \Lambda^{-}=\left[\begin{array}{cc}
I_{\mathbf{l}_{\infty}\left(\mathcal{I}, \mathbf{B}_{2}\right)} & 0 \\
0 & I_{\mathbf{B}}
\end{array}\right]-\left[\begin{array}{cc}
I_{\mathbf{l}_{\infty}\left(\mathcal{I}, \mathbf{B}_{2}\right)}-\mathcal{P}_{Y_{L}} & 0 \\
\mathcal{P}_{Y_{\mathcal{L}}} \ell L^{-} & \mathcal{L} \mathcal{L}^{-}
\end{array}\right] \\
& =\left[\begin{array}{cc}
\mathcal{P}_{Y_{L}} & 0 \\
-\mathcal{P}_{Y_{\mathcal{L}}} \ell L^{-} & I_{\mathbf{B}}-\mathcal{L} \mathcal{L}^{-}
\end{array}\right]=\left[\begin{array}{cc}
\mathcal{P}_{Y_{L}} & 0 \\
-\mathcal{P}_{Y_{\mathcal{L}}} \ell L^{-} & \mathcal{P}_{Y_{\mathcal{L}}}
\end{array}\right] .
\end{aligned}
$$

The operator $\mathcal{P}_{Y_{\Lambda}}$ is indeed a projection, because it has the property $\mathcal{P}_{Y_{\Lambda}}^{2}=\mathcal{P}_{Y_{\Lambda}}$,

$$
\begin{aligned}
& \mathcal{P}_{Y_{\Lambda}}^{2}=\left[\begin{array}{cc}
\mathcal{P}_{Y_{L}} & 0 \\
-\mathcal{P}_{Y_{\mathcal{L}}} \ell L^{-} & \mathcal{P}_{Y_{\mathcal{L}}}
\end{array}\right]\left[\begin{array}{cc}
\mathcal{P}_{Y_{L}} & 0 \\
-\mathcal{P}_{Y_{\mathcal{L}}} \ell L^{-} & \mathcal{P}_{Y_{\mathcal{L}}}
\end{array}\right] \\
& =\left[\begin{array}{cc}
\mathcal{P}_{Y_{L}}^{2} & 0 \\
-\mathcal{P}_{Y_{\mathcal{L}}} \ell L^{-} \mathcal{P}_{Y_{L}}-\mathcal{P}_{Y_{\mathcal{L}}}^{2} \ell L^{-} & \mathcal{P}_{Y_{\mathcal{L}}}^{2}
\end{array}\right]=\left[\begin{array}{cc}
\mathcal{P}_{Y_{L}} & 0 \\
-\mathcal{P}_{Y_{\mathcal{L}}} \ell L^{-} & \mathcal{P}_{Y_{\mathcal{L}}}
\end{array}\right]=\mathcal{P}_{Y_{\Lambda}} .
\end{aligned}
$$

Therefore, the condition $\mathcal{P}_{Y_{\Lambda}} f=\left[\begin{array}{cc}\mathcal{P}_{Y_{L}} & 0 \\ -\mathcal{P}_{Y_{\mathcal{L}}} \ell L^{-} & \mathcal{P}_{Y_{\mathcal{L}}}\end{array}\right]\left[\begin{array}{c}\varphi \\ \alpha\end{array}\right]=0$ is equivalent to the solvability conditions (3) and (6) for the boundary value problem (1), (2). The proof of the theorem is complete.

Remark 5. If the operator equation (1) in the boundary value problem (1), (2) is everywhere solvable, then the projection $\mathcal{P}_{Y_{\Lambda}}$ has the form

$$
\mathcal{P}_{Y_{\Lambda}}=\left[\begin{array}{cc}
0 & 0 \\
-\mathcal{P}_{Y_{\mathcal{L}}} \ell L_{r}^{-1} & \mathcal{P}_{Y_{\mathcal{L}}}
\end{array}\right]
$$

because $\mathcal{P}_{Y_{L}} \equiv 0$ and $L^{-}=L_{r}^{-1}$ in this case. 
Example. Consider the operator equation

$$
(L z)(t):=z(t)-M(t) \int_{0}^{1} N(s) z(s) d s=\varphi(t)
$$

where

$$
M(t)=\operatorname{diag}\left\{e^{t}, e^{t}, \ldots, e^{t}, e^{t}, \ldots\right\}, \quad N(s)=\operatorname{diag}\{s, 0, s, 0, \ldots, s, 0, \ldots\} .
$$

Let the vector function $\varphi(t)$ act from the interval $[0,1]$ into the Banach space $\mathbf{c}$ of all convergent numerical sequences: $\varphi(t) \in \mathbf{C}([0,1], \mathbf{c}):=\{\varphi(\cdot):[0,1] \rightarrow \mathbf{c}\}$; let the operator functions $M(t)$ and $N(t)$ act from the Banach space $\mathbf{C}([0,1], \mathbf{c})$ into itself with the norms

$$
\left\|M \left|\| | _ { \mathbf { C } ( [ 0 , 1 ] , \mathbf { c } ) } = \operatorname { s u p } _ { t \in [ 0 , 1 ] } \| M ( t ) \| _ { \mathbf { c } } , \quad \| \left\|N \left|\left\|\left.\right|_{\mathbf{C}([0,1], \mathbf{c})}=\sup _{t \in[0,1]}\right\| N(t) \|_{\mathbf{c}} .\right.\right.\right.\right.
$$

It follows from the definition of the operator functions $M(t)$ and $N(t)$ that

$$
\||M|\|_{\mathbf{C}([0,1], \mathbf{c})}=\sup _{\substack{t \in[0,1] \\ i, j \in \mathbb{N}}}\left|m_{i j}(t)\right|=\sup _{t \in[0,1]}\left|e^{t}\right| \leq e, \quad\left\||| N\left|\|_{\mathbf{C}([0,1], \mathbf{c})}=\sup _{\substack{t \in[0,1] \\ i, j \in \mathbb{N}}}\right| n_{i j}(t)\left|=\sup _{t \in[0,1]}\right| t \mid \leq 1 .\right.
$$

Then

$$
\begin{aligned}
\|L\|_{\mathbf{C}([0,1], \mathbf{c})} & =\sup _{z \in \mathbf{C}([0,1], \mathbf{c})} \frac{\|L z\|_{\mathbf{C}([0,1], \mathbf{c})}}{\|z\|_{\mathbf{C}([0,1], \mathbf{c})}}=\sup _{z \in \mathbf{C}([0,1], \mathbf{c})} \frac{\left\|z(t)+M(t) \int_{0}^{1} N(s) z(s) d s\right\|_{\mathbf{C}([0,1], \mathbf{c})}}{\|z\|_{\mathbf{C}([0,1], \mathbf{c})}} \\
& \leq \sup _{z \in \mathbf{C}_{z \neq 0,1], \mathbf{c})}} \frac{\|z(t)\|_{\mathbf{C}([0,1], \mathbf{c})}+\|M(t)\|_{\mathbf{C}([0,1], \mathbf{c})} \int_{0}^{1}\|N(s)\|_{\mathbf{C}([0,1], \mathbf{c})}\|z(s)\|_{\mathbf{C}([0,1], \mathbf{c})} d s}{\|z\|_{\mathbf{C}([0,1], \mathbf{c})}} \\
& \leq \sup _{z \in \mathbf{C}([0,1], \mathbf{c})} \frac{(1+e)\|z(t)\|_{\mathbf{C}([0,1], \mathbf{c})}}{\| z 0} \leq 1+e .
\end{aligned}
$$

Therefore, the operator $L$ is a linear bounded operator acting from the Banach space $\mathbf{C}([0,1], \mathbf{c})$ of continuous functions on the interval $[0,1]$ into itself.

Let us find solutions of Eq. (17) satisfying the conditions

$$
\ell z(\cdot)=\int_{0}^{1} S(t) z(t) d t=\alpha
$$

where

$$
\begin{aligned}
& S(t)=\operatorname{diag}\left\{S_{(2 \times 4)}(t), S_{(2 \times 4)}(t), \ldots\right\}, \quad S_{(2 \times 4)}(t)=\left(\begin{array}{cccc}
e^{-t} & 0 & e^{-t} & 0 \\
(2 t-1) e^{-t} & 0 & 0 & 0
\end{array}\right), \\
& \||S|\|_{\mathbf{C}([0,1], \mathbf{c})}=\sup _{t \in[0,1]}\|S(t)\|_{\mathbf{c}} .
\end{aligned}
$$

The vector functional $\ell$ acts from the space $\mathbf{C}([0,1], \mathbf{c})$ into the Banach space $\mathbf{c}$ and is bounded, $\alpha \in \mathbf{c}, \alpha=\operatorname{col}\left(\alpha_{1}, \alpha_{2}, \alpha_{3}, \ldots\right)$. 
For the operator $L$, the bounded projections $\mathcal{P}_{N(L)}$ and $\mathcal{P}_{Y_{L}}$ and the operators $\overline{\mathcal{P}}_{Y_{L}}$ and $\overline{\mathcal{P}}_{N(L)}$ acquire the form

$$
\begin{aligned}
\left(\mathcal{P}_{N(L)} z\right)(t) & =X(t) \int_{0}^{1} \Gamma(s) z(s) d s, \quad\left(\mathcal{P}_{Y_{L}} y\right)(t)=\Psi \int_{0}^{1} \Phi(s) y(s) d s \\
\left(\overline{\mathcal{P}}_{Y_{L}} z\right)(t) & =\Psi \int_{0}^{1} \Gamma(s) z(s) d s, \quad\left(\overline{\mathcal{P}}_{N(L)} y\right)(t)=X(t) \int_{0}^{1} \Phi(s) y(s) d s
\end{aligned}
$$

respectively, where

$$
\begin{aligned}
X(t) & =\operatorname{diag}\left\{X_{(4 \times 2)}(t), X_{(4 \times 2)}(t), \ldots\right\}, \quad \Gamma(t)=\operatorname{diag}\left\{\Gamma_{(2 \times 4)}(t), \Gamma_{(2 \times 4)}(t), \ldots\right\}, \\
X_{(4 \times 2)}(t) & =\left(\begin{array}{cccc}
e^{t} & 0 & 0 & 0 \\
0 & 0 & e^{t} & 0
\end{array}\right)^{\mathrm{T}}, \quad \Gamma_{(2 \times 4)}(t)=\left(\begin{array}{cccc}
t & 0 & 0 & 0 \\
0 & 0 & t & 0
\end{array}\right), \\
\Phi(t) & =\operatorname{diag}\left\{\Phi_{(2 \times 4)}(t), \Phi_{(2 \times 4)}(t), \ldots\right\}, \quad \Psi(t)=\operatorname{diag}\left\{\Psi_{(4 \times 2)}(t), \Psi_{(4 \times 2)}(t), \ldots\right\}, \\
\Phi_{(2 \times 4)}(t) & =\left(\begin{array}{llll}
t & 0 & 0 & 0 \\
0 & 0 & t & 0
\end{array}\right), \quad \Psi_{(4 \times 2)}(t)=\left(\begin{array}{llll}
2 & 0 & 0 & 0 \\
0 & 0 & 2 & 0
\end{array}\right)^{\mathrm{T}} .
\end{aligned}
$$

The boundedness of the projections $\mathcal{P}_{N(L)}$ and $\mathcal{P}_{Y_{L}}$ implies the generalized invertibility and hence the normal solvability of the operator $L$. Therefore, the original equation (17) is solvable if and only if the vector function $\varphi(t)$ satisfies the condition

$$
\left(\mathcal{P}_{Y_{L}} \varphi\right)(t)=\Psi \int_{0}^{1} \Phi(s) \varphi(s) d s=0
$$

which is satisfied if and only if the components of the vector function $\varphi(t)=\operatorname{col}\left(\varphi_{1}(t), \varphi_{2}(t)\right.$, $\left.\varphi_{3}(t), \ldots\right)$ satisfy the relations

$$
\int_{0}^{1} s \varphi_{2 k-1}(s) d s=0, \quad k=1,2,3, \ldots
$$

Under condition (19), the operator equation (17) has the solution [11, p. 82; 13, p. 69; 31]

$$
z(t)=\left(\mathcal{P}_{N(L)} \hat{z}\right)(t)+\left(L^{-} \varphi\right)(t)=X(t) \int_{0}^{1} \Gamma(s) \hat{z}(s) d s+\varphi(t)+M_{1}(t) \int_{0}^{1} N_{1}(s) \varphi(s) d s
$$

where $\hat{z}(t)$ is an arbitrary element of the space $\mathbf{C}([0,1]$, $\mathbf{c})$ and

$$
\begin{array}{rlrl}
M_{1}(t) & =\operatorname{diag}\left\{M_{(2 \times 4)}(t), M_{(2 \times 4)}(t), \ldots\right\}, & & N_{1}(s)=\operatorname{diag}\left\{N_{(4 \times 2)}(s), N_{(4 \times 2)}(s), \ldots\right\}, \\
M_{(2 \times 4)}(t) & =\left(\begin{array}{cccc}
2\left(e^{t}-1\right) & 0 & -e^{t} & -e^{t} \\
0 & e^{t} & 0 & 0
\end{array}\right), & N_{(4 \times 2)}(s)=\left(\begin{array}{cccc}
s & 0 & s & s \\
0 & 0 & 0 & 0
\end{array}\right)^{\mathrm{T}} .
\end{array}
$$

By substituting the general solution (20) into the boundary condition (18), we obtain the operator equation

$$
\mathcal{L} \hat{z}+\ell\left(L^{-} \varphi\right)(\cdot)=\alpha
$$


where

$$
(\mathcal{L} \hat{z})(\cdot)=\left(\ell \mathcal{P}_{N(L)} \hat{z}\right)(\cdot)=\int_{0}^{1} S(t) X(t) \int_{0}^{1} \Gamma(s) \hat{z}(s) d s d t=Q \int_{0}^{1} \Gamma(s) \hat{z}(s) d s .
$$

The linear bounded matrix operator

$$
Q=\int_{0}^{1} S(t) X(t) d t=\operatorname{diag}\left\{\left(\begin{array}{ll}
1 & 1 \\
0 & 0
\end{array}\right),\left(\begin{array}{ll}
1 & 1 \\
0 & 0
\end{array}\right), \ldots\right\}
$$

acts from the Banach space $\mathbf{c}$ of bounded sequences converging to a finite limit into itself $(Q: \mathbf{c} \rightarrow \mathbf{c})$ and is generalized-invertible.

By setting $\hat{z}_{0}=\int_{0}^{1} \Gamma(s) \hat{z}(s) d s$, we rewrite the operator equation (21) in the form of the matrix operator equation

$$
Q \hat{z}_{0}=\alpha-\ell \varphi(\cdot)-\ell M_{1}(\cdot) \int_{0}^{1} N_{1}(s) \varphi(s) d s .
$$

By constructing the projection $\mathcal{P}_{Y_{Q}}: \mathbf{c} \rightarrow Y_{Q}$ :

$$
\mathcal{P}_{Y_{Q}}=\operatorname{diag}\left\{\left(\begin{array}{ll}
0 & 0 \\
0 & 1
\end{array}\right),\left(\begin{array}{ll}
0 & 0 \\
0 & 1
\end{array}\right), \ldots\right\},
$$

we find that Eq. (22) is solvable if and only if

$$
\mathcal{P}_{Y_{Q}}\left\{\alpha-\ell \varphi(\cdot)-\ell M_{1}(\cdot) \int_{0}^{1} N_{1}(s) \varphi(s) d s\right\}=0 .
$$

After transformations, we find that the components of the vector $\alpha \in \mathbf{c}$ and the vector function $\varphi(t) \in \mathbf{C}([0,1], \mathbf{c})$ should satisfy the conditions

$$
\alpha_{2 k}-\int_{0}^{1}(2 s-1) e^{-s} \varphi_{4 k-3}(s) d s-\frac{6-2 e}{e} \int_{0}^{1} s \varphi_{8 k-7}(s) d s=0, \quad k=1,2,3, \ldots
$$

Under these conditions, the operator equation (22) has the solution

$$
\hat{z}_{0}=\mathcal{P}_{N(Q)} c+Q^{-}\left\{\alpha-\ell \varphi(\cdot)-\ell M_{1}(\cdot) \int_{0}^{1} N_{1}(s) \varphi(s) d s\right\}
$$

where $c$ is an arbitrary element of the space $\mathbf{c}$ and

$$
\mathcal{P}_{N(Q)}=\operatorname{diag}\left\{\left(\begin{array}{cc}
1 & 0 \\
-1 & 0
\end{array}\right),\left(\begin{array}{cc}
1 & 0 \\
-1 & 0
\end{array}\right), \ldots\right\}, \quad Q^{-}=\operatorname{diag}\left\{\left(\begin{array}{ll}
0 & 0 \\
1 & 0
\end{array}\right),\left(\begin{array}{ll}
0 & 0 \\
1 & 0
\end{array}\right), \ldots\right\} .
$$

By substituting the expression (24) for $\hat{z}_{0}$ for $\int_{0}^{1} \Gamma(s) \hat{z}(s) d s$ into (20), we obtain the general solution of the boundary value problem (17), (18) in the form

$$
\begin{aligned}
z(t)= & X(t) \mathcal{P}_{N(Q)} c+\varphi(t)-X(t) Q^{-} \ell \varphi(\cdot) \\
& -X(t) Q^{-} \ell M_{1}(\cdot) \int_{0}^{1} N_{1}(s) \varphi(s) d s+M_{1}(t) \int_{0}^{1} N_{1}(s) \varphi(s) d s+X(t) Q^{-} \alpha .
\end{aligned}
$$

DIFFERENTIAL EQUATIONS Vol. 50 No. $3 \quad 2014$

(Reg. No. 305, 19.3.2014) 
Therefore, the boundary value problem (17), (18) is solvable if and only if conditions (19) and (23) are satisfied, and, in this case, it has the family of solutions

$$
\begin{aligned}
z(t)= & X(t) \mathcal{P}_{N(Q)} c+\left[\varphi(t)-X(t) Q^{-} \ell \varphi(\cdot)\right] \\
& +\left[M_{1}(t)-X(t) Q^{-} \ell M_{1}(\cdot)\right] \int_{0}^{1} N_{1}(s) \varphi(s) d s+X(t) Q^{-} \alpha .
\end{aligned}
$$

\section{REFERENCES}

1. Lyapunov, A.M., Obshchaya zadacha ob ustoichivosti dvizheniya (General Problem of the Stability of Motion), Moscow: Gosudarstv. Izdat. Tekhn.-Teor. Lit., 1950.

2. Malkin, I.G., Nekotorye zadachi teorii nelineinykh kolebanii (Some Problems of the Theory of Nonlinear Oscillations), Moscow: Gosudarstv. Izdat. Tehn.-Teor. Lit., 1956.

3. Grebennikov, E.A. and Ryabov, Yu.A., Konstruktivnye metody analiza nelineinykh sistem (Constructive Methods for the Analysis of Nonlinear Systems), Moscow, 1979.

4. Hale, J., Theory of Functional Differential Equations, New York: Springer, 1977. Translated under the title Teoriya funktsional'no-differentsial'nykh uravnenii, Moscow: Mir, 1984.

5. Samoilenko, A.M. and Perestyuk, N.A., Differentsial'nye uravneniya s impul'snym vozdeistviem (Impulsive Differential Equations), Kiev, 1987.

6. Tvrdy, M. and Veivoda, O., General Boundary Value Problems for an Integrodifferential System and Its Adjoint, C̆as. prestov. mat., 1973, vol. 98, pp. 26-42.

7. Schwabic, S., Tvrdy, M., and Veivoda, O., Differential and Integral Equations. Boundary Value Problems and Adjoints, Praga, 1979.

8. Mawhin, J., Topological Degree Methods in Nonlinear Boundary Value Problems, Regional conference series in mathematics. Providence, 1979, no. 40.

9. Ben-Israel, A. and Greville, T.N.E., Generalized Inverse Theory and Applications, New York, 2003, 2nd ed.

10. Boichuk, A.A., Konstruktivnye metody analiza kraevykh zadach (Constructive Methods in the Analysis of Boundary Value Problems), Kiev: Naukova Dumka, 1990.

11. Boichuk, A.A., Zhuravlev, V.F., and Samoilenko, A.M., Obobshchenno-obratnye operatory $i$ neterovy kraevye zadachi (Generalized Inverse Operators and Fredholm Boundary Value Problems), Kiev: Nats. Akad. Nauk Ukraini, 1995.

12. Boichuk, A.A., Zhuravlev, V.F., and Samoilenko, A.M., Fredholm Boundary Value Problems for Impulsive Delay Differential Systems, Differ. Uravn., 1994, vol. 30, no. 10, pp. 1677-1682.

13. Boichuk, A.A. and Samoilenko, A.M., Generalized Inverse Operators and Fredholm Boundary-Value Problems, Utrecht: VSP, 2004.

14. Daletskii, Yu.L. and Krein, M.G., Ustoichivost' reshenii differentsial'nykh uravnenii v banakhovom prostranstve (Stability of Solutions of Differential Equations in a Banach Space), Moscow: Nauka, 1970.

15. Boichuk, O.A. and Panasenko, E.V., Boundary Value Problems for Differential Equations in a Banach Space, Neliniini Koliv., 2009, vol. 12, no. 1, pp. 16-19.

16. Samoilenko, A.M. and Teplins'kii, Yu.V., Elementi matematichnö̈ teorï̈ evolyutsiinikh rivnyan' u banakhovikh prostorakh (Elements of the Mathematical Theory of Evolution Equations in Banach Spaces), Kiev: Nats. Akad. Nauk Ukraini, 2008.

17. Boichuk, A. and Pokutnij, A., Bounded Solutions of Linear Perturbed Differential Equations in a Banach Space, Tatra Mt. Math. Publ., 2007, vol. 39, pp. 1-12.

18. Krein, S.G., Lineinye uravneniya $v$ banakhovom prostranstve (Linear Equations in a Banach Space), Moscow: Nauka, 1971.

19. Samoilenko, A.M., Boichuk, A.A., and Krivosheya, S.A., Boundary-Value Problem for Linear Systems of Integrodifferential Equations with Degenerate Kernel, Ukr. Mat. Zh., 1996, vol. 48, no. 11, pp. 15761579.

20. Samoilenko, A.M., Shkil', M.I., and Yakovec', V.P., Linear Systems of Differential Equations with Singularities, Kyiv, 2000.

21. Chistyakov, V.F. and Shcheglova, A.A., Izbrannye glavy teorii algebro-differentsial'nykh sistem (Selected Chapters in the Theory of Algebraic-Differential Systems), Novosibirsk: Nauka, 2003. 
22. Boichuk, A.A. and Shegda, L.M., Bifurcation of Solutions of Singular Fredholm Boundary Value Problems, Differ. Equat., 2011, vol. 47, no. 4, pp. 453-461.

23. Khelemskii, A.Ya., Lektsii po funktsional'nomu analizu (Lectures on Functional Analysis), Moscow, 2004.

24. Gokhberg, I.Ts. and Krupnik, N.Ya., Vvedenie v teoriyu odnomernykh singulyarnykh integral'nykh operatorov (Introduction to the Theory of One-Dimensional Singular Integral Operators), Kishinev: Stiinca, 1973.

25. Popov, M.M., Complemented Subspaces and Some Problems of the Modern Geometry of Banach Spaces, Mat. S'godni, 2007, vol. 13, pp. 78-116.

26. Fredholm, I., Sur une classe d'equations fonctionnelles, Acta Math., 1903, vol. 27, pp. 265-390.

27. Noether, F., Über eine Klasse singulärer Integralgleichungen, Math. Ann., 1921, no. 82, pp. 42-63.

28. Korolyuk, V.S. and Turbin, A.F., Matematicheskie osnovy fazovogo ukrupneniya slozhnykh sistem (Mathematical Foundations of the State Lumping of Complex Systems), Kiev: Naukova Dumka, 1978.

29. Abdullaev, A.R. and Burmistrova, A.B., Topological Fredholm Operators: Generalized Invertibility and Additive Representation, Izv. Vyssh. Uchebn. Zaved. Mat., 1994, no. 6, pp. 3-7.

30. Generalized Inverses and Applications, Nashed, M.Z., Ed., New York, 1976.

31. Zhuravlev, V.F., Solvability Criterion and Representation of Solutions of $n$-Normal and $d$-Normal Linear Operator Equations in a Banach Space, Ukr. Mat. Zh., 2010, vol. 62, no. 2, pp. 167-182.

32. Wexler, D., On Boundary Value Problems for an Ordinary Linear Differential System, Ann. di Mat. pura et Appl., 1968, vol. 80, pp. 122-136. 